\title{
Extraocular muscle enlargement: rare presentation of plasmablastic lymphoma
}

\author{
Mridula Jacob, Vijaya Pai H. \\ Department of Opthalmology, Katsurba Medical College, Manipal Academy of \\ Higher Education, Manipal, India
}

\begin{abstract}
Plasmablastic lymphoma (PBL) is a very rare and highly aggressive variant of non-Hodgkin lymphoma. It is usually seen in the oral cavity of human immunodeficiency virus (HIV)-affected individuals. Very few cases of PBL are reported in the orbit till date. Morphologically and immunologically, it can mimic plasma cell myeloma. It is highly fatal and poses diagnostic and therapeutic challenges to the treating clinician. This scenario makes reporting of such rare tumors more relevant. We report a rare case of PBL of the orbit in a 49-year-old HIV-positive lady who presented with acute onset of painful proptosis and loss of vision in her left eye.
\end{abstract}

Keywords: extraocular muscle enlargement, HIV, NHL, plasmablastic lymphoma

\section{Introduction}

Orbital lymphoma is a type of non-Hodgkin lymphoma (NHL) that can involve the conjunctiva, lacrimal gland, eyelid, or extraocular muscles. ${ }^{1}$ It can be a primary lymphoma when it arises from the orbital tissues itself or a secondary lymphoma when there is a metastatic spread from an extraorbital site. Orbital lymphomas are very rare and form about $1 \%$ of all $\mathrm{NHL}$ and $55 \%$ of all primary orbital tumors.,3 It is more commonly seen in immunocompromised patients. But there are case reports of orbital lymphoma in human immunodeficiency virus (HIV)-negative otherwise healthy individuals also. ${ }^{3}$ Majority of the cases are extranodal marginal zone B-cell lymphomas of mucosa-associated lymphoid tissue type. ${ }^{3}$ Here, we are reporting a very rare, fatal variant of orbital lymphoma plasmablastic type, which is usually seen in the oral cavity of HIV-positive individuals. ${ }^{4}$

\section{Case description}

A 49-year-old immunocompromised female patient presented with an acute onset of painful proptosis of the left eye associated with loss of vision of 7 days duration. There was no history of trauma and fever. She was diagnosed to be HIV positive 5 years ago but was not on any antiretroviral therapy at the time of presentation.

Correspondence: Vijaya Pai H., Department of Opthalmology, Katsurba Medical College, Manipal Academy of Higher Education, Manipal, India.

E-mail: paivijaya@yahoo.co.in 


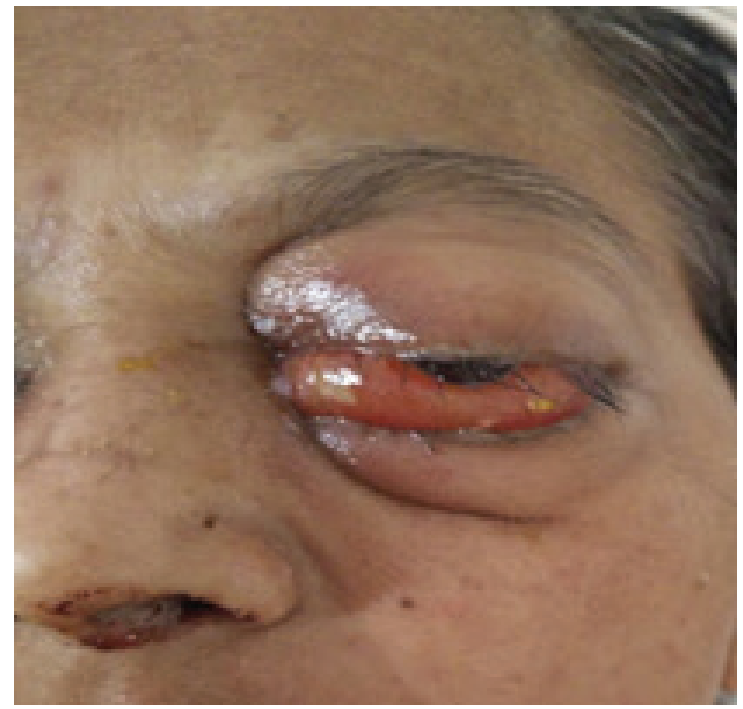

Fig. 1. Left eye proptosis with lid edema, conjunctival chemosis.

On examination, the best corrected visual acuity was $6 / 6 p$ Snellen's in the right eye and counting finger $1 \mathrm{~m}$ in the left eye. Right eye examination was normal. Left eye showed proptosis of $9 \mathrm{~mm}$ on Hertel exophthalmometry. There was lid edema, conjunctival congestion, chemosis, and exposure keratitis (Fig. 1). Extraocular movements were restricted in all directions. Pupil showed relative afferent pupillary defect. Fundus showed pale disc with hemorrhages and cotton wool spots superior to disc.

A provisional diagnosis of orbital cellulitis was made. Patient was started on intravenous antibiotics, amoxicillin with clavulanic acid combination $(1.2 \mathrm{~g}$ thrice daily), gentamicin ( $80 \mathrm{mg}$ twice daily), and tinidazole ( $800 \mathrm{mg}$ once daily). Her hemoglobin was $6.9 \mathrm{~g} / \mathrm{dl}$, erythrocyte sedimentation rate was $109 \mathrm{~mm} /$ hour (Ves-Matic cube method), CD4/CD8 ratio was 0.11 (normal value 2); absolute CD4 count was 91.74 cells/ $\mu$ l (normal value 500-1,200 cells/ $\mu \mathrm{l}$ ), lactate dehydrogenase (LDH) was highly raised: 959 IU/I (normal value 105-333 IU/I). Reports came positive for HIV-1. Computed tomography (CT) scan of the orbit showed enlargement of all the extraocular muscles of the left eye, with swollen bulky medial rectus abutting optic nerve at the orbital apex. Right medial rectus was also enlarged. There was no evidence of mass lesion or bony destruction (Fig. 2). Thyroid function test was done in view of enlarged muscles on $\mathrm{CT}$ and was normal. Chest $\mathrm{x}$-ray was normal. Ultrasound abdomen did not 
show any hepatosplenomegaly or enlarged lymph nodes. Bone marrow study was normal.

Transnasal endoscopic incision biopsy of the enlarged medial rectus of the left eye was done. Histopathology showed monomorphic population of medium sized to large abnormal lymphoid cells with round nuclei, some with 2-5 nucleoli and irregular nuclear membrane, others with eccentric nuclei with single nucleoli, moderate cytoplasm with perinuclear hof imparting a plasmacytoid appearance along with brisk mitosis, interspersed mature lymphocytes, apoptotic bodies, and numerous triple body macrophages containing karyorrhectic debris focally imparting a starry sky appearance (Fig. 3). Immunohistochemically, tumor cells were CK, CD79a, CD20, CD3 negative and CD138 positive.

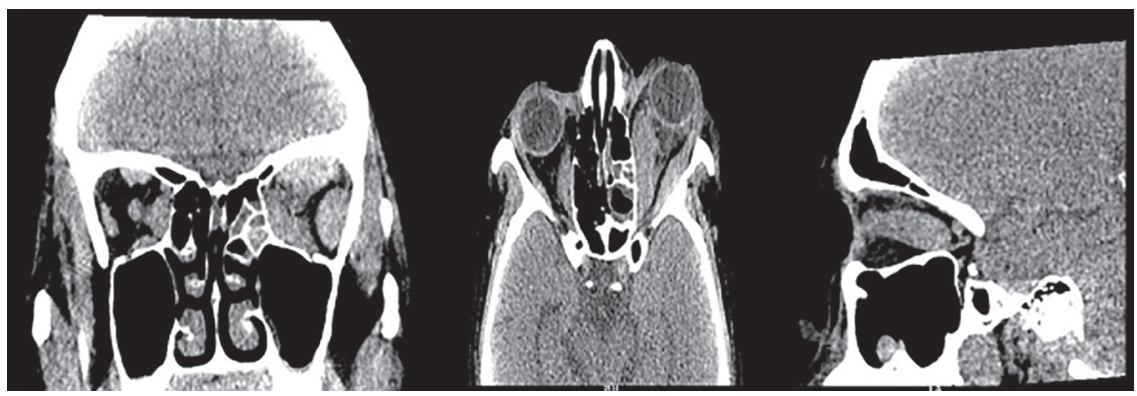

Fig. 2. CT images of orbit showing bulky extraocular muscles of left eye and enlarged medial rectus of right eye with no mass lesion.

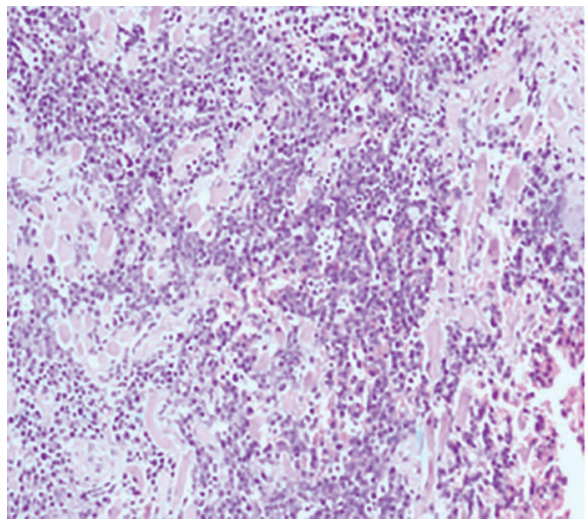

Infiltration of tumor cells into and between skeletal muscle

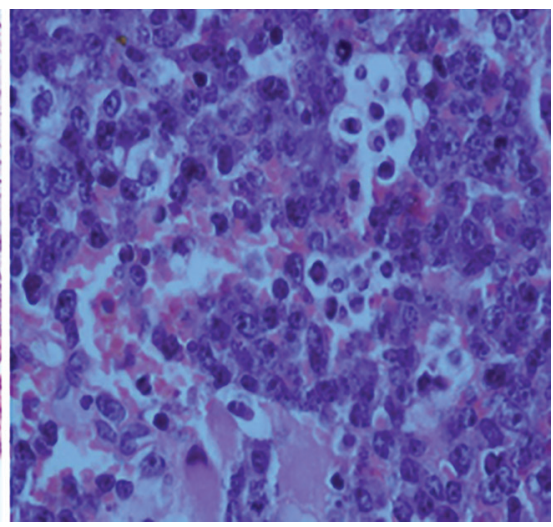

Medium sized to large abnormal lymphoid cells

Fig. 3. Histopathology images. 
These features confirmed the diagnosis of high-grade NHL plasmablastic type. Patient was referred to medical oncology. She was restarted on antiretroviral therapy; however, she refused further chemotherapy.

\section{Discussion}

Plasmablastic lymphoma (PBL) is considered as a distinct subtype of diffuse large B-cell lymphoma, which is seen more commonly in patients with HIV infection. ${ }^{1}$ In 2008, Valenzuela et al. reported a case of PBL in a HIV-positive female, wherein the patient had lesions in the oral cavity following which she developed orbital manifestations, ${ }^{5}$ and also by Barkhuysen et al., in which the presentation of PBL exactly mimicked orbital cellulitis. ${ }^{6}$ Ocular involvement of PBL is very rare, with very few cases having been reported in literature till now. ${ }^{2}$ Majority of the patients with PBL present with diminution of vision and/or proptosis of the affected eye. Conjunctival chemosis, lid swelling, ptosis, and loss of sensation along the trigeminal nerve are other findings described. Mild to severe globe motility restriction is a consistent finding on examination. ${ }^{2}$ Our patient too had these features. Clinically, orbital inflammation is the most common differential diagnosis. This reflects the challenge posed to the physician in coming to a proper diagnosis and specifies the importance of histopathological analysis of the involved tissue.

In most of the cases of PBL reported in literature, patients had mass lesion in the orbit on CT. Our patient had massive enlargement of all the extraocular muscles of the left eye and medial rectus of the right eye. None of the cases of PBL reported in literature had such a presentation. Discrete lymphomatous lesions of the extraocular muscles are rare. They are often low-grade malignancies that respond well to radiotherapy and have a good prognosis. ${ }^{7}$ However, our patient had the aggressive type of NHL. Clinically, there was no involvement of the right eye though CT scan showed enlargement of right medial rectus. At the time of presentation, metastatic involvement of liver, bone marrow, or lymph nodes is not uncommon, showing the highly aggressive nature of PBL. Our patient did not show any evidence of systemic involvement. In view of bilateral orbital involvement without any systemic spread, she was considered to be Ann Arbor stage $\|_{E \text {. }}$

Diagnosis of PBL is confirmed by histopathology and immunohistochemical studies, which show large immunoblasts or plasma cells that express plasma cell markers (CD138, CD38, CD79a) and lack B-cell markers(CD19, CD20). In our patient, $\mathrm{CD} 138$ was positive. The main challenge in the diagnosis of $\mathrm{PBL}$ is its morphologic and immunologic resemblance to plasma cell myeloma. ${ }^{9}$ Differentiation is mainly with the help of immunohistochemical markers. Though Epstein-Barr virus (EBV) infection was invariably noticed in most of the cases reported in literature, ${ }^{2,5}$ we could not perform any tests for EBV as this facility was not available in our lab. 
The International Prognostic Index scoring system is the most commonly used risk stratification tool for aggressive lymphomas, and it includes age, performance status, LDH levels, number of extranodal sites involved, and clinical stage to prognostic survival. ${ }^{8}$ According to this, we categorized our patient under high-intermediate risk group.

Though orbital involvement in PBL is very rare, its incidence is on the rise in HIV-positive individuals. This is because of the increased awareness about PBL in clinicians and the pathologists. ${ }^{8}$ Initial clinical presentation can resemble orbital inflammation. But when this type of presentation coincides with an immunocompromised status, the clinician should always keep PBL as one of the differential diagnosis. Ocular adnexal lymphoma with discrete involvement of extraocular muscles is rare. Our patient had unusual features of extraocular muscle enlargement as found in $\mathrm{CT}$, which has not been reported in the literature for PBL till date.

\section{References}

1. Christopher MN, Christopher DJ, Andrew JN, et al. Primary orbital lymphoma. Hematol J. 2002;3:14-16.

2. Mulay K, Ali MJ, Reddy VA, et al. Orbital plasmablastic lymphoma: a clinic pathological correlation of a rare disease \& review of literature. Clin Ophthalmol. 2012;6:2049-2057.

3. Eckardt AM, Lemound J, Rana M, Gellrich N-C. Orbital lymphoma: Diagnostic approach and treatment outcome. World J Surg Oncol. 2013;11:73.

4. Corti M, Minué G, Campitelli A, et al. An aggressive plasmablastic lymphoma of the oral cavity as primary manifestation of acquired immunodeficiency syndrome: case report and literature review. Int Arch Otorhinolaryngol. 2015;19:354-358.

5. Valenzuela AA, Walker NJ, Sullivan TJ, et al. Plasmablastic lymphoma in the orbit - case report. Orbit. 2008;27:227-229.

6. Barkhuysen R, Merkx MA, Weijs WL, et al. Plasmablastic lymphoma mimicking orbital cellulitis. Oral Maxillofac Surg. 2008;12:125.

7. Watkins LM, Carter KD, Nerad JA. Ocular adnexal lymphoma of the extraocular muscles: case series from the University of lowa and review of the literature. Ophthal Plast Reconstr Surg. 2011;27:471-476.

8. Castillo JJ, Bibas M, Miranda RN. The biology and treatment of plasmablastic lymphoma. Blood. 2015;125(15):2323-2330.

9. Veqa F, Chanq CC, Medeiros LJ, et al. Plasmablastic lymphomas and plasmablastic plasma cell myelomas have nearly identical immunophenotypic profiles. Mod Pathol. 2005;18:873. 